\title{
EFFICIENT POWER ALLOCATION USING GRAPH NEURAL NETWORKS AND DEEP ALGORITHM UNFOLDING
}

\author{
Arindam Chowdhury ${ }^{\star}$, Gunjan Verma ${ }^{\dagger}$, Chirag Rao ${ }^{\dagger}$, Ananthram Swami ${ }^{\dagger}$, and Santiago Segarra \\ ${ }^{\star}$ Rice University, USA \\ †US Army’s CCDC Army Research Laboratory, USA
}

\begin{abstract}
We study the problem of optimal power allocation in a single-hop ad hoc wireless network. In solving this problem, we propose a hybrid neural architecture inspired by the algorithmic unfolding of the iterative weighted minimum mean squared error (WMMSE) method, that we denote as unfolded WMMSE (UWMMSE). The learnable weights within UWMMSE are parameterized using graph neural networks (GNNs), where the time-varying underlying graphs are given by the fading interference coefficients in the wireless network. These GNNs are trained through a gradient descent approach based on multiple instances of the power allocation problem. Once trained, UWMMSE achieves performance comparable to that of WMMSE while significantly reducing the computational complexity. This phenomenon is illustrated through numerical experiments along with the robustness and generalization to wireless networks of different densities and sizes.
\end{abstract}

Index Terms - Wireless network, power allocation, WMMSE, graph neural network, algorithm unfolding

\section{INTRODUCTION}

Power and bandwidth are fundamental resources that determine the effective capacity of a wireless channel [1]. Hence, optimal allocation of these resources under randomly varying channel characteristics and user demands is essential for the smooth operation of wireless systems. In particular, power allocation in a wireless ad hoc network is crucial to mitigate multi-user interference, one of the main performance-limiting factors. Mathematically, power allocation can be formulated as the problem of optimizing a certain system-level utility function (such as sum rate or harmonic rate) subject to resource budget constraints. Despite the remarkable success of this paradigm [2], many of the associated optimization problems are non-convex and NP-hard [3, 4]. For the canonical case of sumrate maximization, the most commonly used algorithm is weighted minimum mean squared error (WMMSE) minimization [5]. In spite of being close to optimal in practice, it has high computational complexity and slow convergence. This has led the research community to look for faster, data-driven solutions for power allocation.

Deep learning based methods have emerged as promising alternatives to classical methods for resource allocation in recent

Research was sponsored by the Army Research Office and was accomplished under Cooperative Agreement Number W911NF-19-2-0269. The views and conclusions contained in this document are those of the authors and should not be interpreted as representing the official policies, either expressed or implied, of the Army Research Office or the U.S. Government. The U.S. Government is authorized to reproduce and distribute reprints for Government purposes notwithstanding any copyright notation herein.

Emails: \{ac131, segarra\}@rice.edu, \{gunjan.verma.civ, chirag.r.rao.civ, ananthram.swami.civ\}@mail.mil. years [6]. In supervised settings, deep neural networks learn to mimic established classical methods from solved instances [7]. In contrast, some works have followed an unsupervised approach, where one parameterizes the power allocation function using a neural network and directly employs the optimization objective as a loss function, bypassing the need for solved problem instances [8, 9]. While such a procedure is computationally simple, no prior knowledge (e.g., based on classical optimization techniques) is leveraged to inform the algorithm's architectural or hyper-parameter choices.

We advocate a third direction that is unsupervised as no solved instances of the power allocation problem are needed for training, while imitating classical methods by incorporating part of their structure into the layered architecture of the neural network. The goal is to leverage the theoretical models developed with expert knowledge and achieve near-optimal performance with significantly reduced execution time. To accomplish this, we follow the paradigm of algorithm unfolding [10, 11]. The idea is to unfold the iterations of a classical iterative algorithm as a cascade of layers, where each layer has the same update structure as the original algorithm but the parameters can be learned from data. A standard method of unfolding is to parameterize the function of interest using multi-layer perceptrons (MLPs) or convolutional neural networks (CNNs) [12]. However, MLPs and CNNs are not quite suitable for problems in wireless communication. In particular, their performance degrades dramatically when the network size becomes large since MLPs and $\mathrm{CNNs}$ cannot exploit the underlying network topology. We adopt an alternative direction [13 -15], where graph neural networks (GNNs) [16-22] are used to parameterize the power allocation function, thus leveraging the natural representation of wireless networks as graphs. GNNs utilize the structural relationships between nodes to locally process instantaneous channel state information. In this context, we propose an unfolded weighted minimum mean squared error (UWMMSE) method, which is to the best of our knowledge the first GNN-based deep unfolded architecture based on the iterative WMMSE algorithm. UWMMSE simultaneously achieves stateof-the-art performance in utility maximization and computational efficiency for power allocation in wireless networks.

Contribution. The contributions of this paper are twofold:

i) We propose an unfolded version of WMMSE for power allocation in wireless networks, where the learnable modules are parameterized through GNNs.

ii) We empirically illustrate the performance of the proposed method, compare it with state-of-the-art alternatives, and demonstrate its generalization to networks of unseen sizes and densities.

\section{SYSTEM MODEL AND PROBLEM FORMULATION}

We consider a single-hop ad hoc interference network having $M$ distinct single-antenna transceiver pairs. Transmitters are denoted by 
$i$ and the $i$-th transmitter is associated with a single receiver denoted by $r(i)$ for $i \in\{1, \ldots, M\}$. Further, denoting the signal transmitted by $i$ as $x_{i} \in \mathbb{R}$, the received signal $y_{i} \in \mathbb{R}$ at $r(i)$ is given by

$$
y_{i}=h_{i i} x_{i}+\sum_{j=1 \mid j \neq i}^{M} h_{i j} x_{j}+n_{i}
$$

where $h_{i i} \in \mathbb{R}$ is the channel between the $i$-th transceiver pair, $h_{i j} \in$ $\mathbb{R}$ for $i \neq j$ represents the interference between transmitter $j$ and receiver $r(i)$, and $n_{i} \sim \mathcal{N}\left(0, \sigma^{2}\right)$ represents the additive channel noise. We consider time-varying channel states $h_{i j}(t)$, which are stored in a channel-state matrix $\mathbf{H}(t) \in \mathbb{R}^{M \times M}$ where $[\mathbf{H}(t)]_{i j}=$ $h_{i j}(t)$. We can interpret $\mathbf{H}(t)$ as the weighted adjacency matrix of a directed graph with $M$ nodes, where node $i$ represents the $i$-th transceiver pair. The instantaneous data rate $c_{i}$ achievable at receiver $r(i)$ is given by Shannon's capacity theorem

$$
c_{i}(\mathbf{p}(t), \mathbf{H}(t))=\log _{2}\left(1+\frac{h_{i i}^{2}(t) p_{i}(t)}{\sigma^{2}+\sum_{j \neq i} h_{i j}^{2}(t) p_{j}(t)}\right),
$$

where $p_{i}(t) \geq 0$ is the power allocated to transmitter $i$ at time $t$ and $\mathbf{p}(t)=\left[p_{1}(t), \ldots, p_{M}(t)\right]^{\top}$. The objective is to determine the instantaneous power allocation vector $\mathbf{p}(t)$ that maximizes a network utility that is a function of data rates $c_{i}$. Omitting the explicit dependence on $t$ to simplify notation, we formalize the standard sum-rate maximization problem under power constraints as follows

$$
\max _{\mathbf{p}} \sum_{i=1}^{M} c_{i}(\mathbf{p}, \mathbf{H}) \quad \text { s.t. } 0 \leq p_{i} \leq p_{\max } \text {, for all } i
$$

where $p_{\max }$ denotes the maximum available power at every transmitter. Though seemingly simple, this optimization problem has been shown to be NP-hard [3 23]. In this work, we aim to achieve an effective and efficient solution to (3), where $\mathbf{H}$ is drawn from an $a c$ cessible distribution $\mathcal{H}$. Here, by effective we mean a solution that achieves performance close to that of a near-optimal classical approach, while accessible distribution means that either $\mathcal{H}$ is known or we can easily sample from it.

Classical approaches focus on finding approximate solutions for a single instance of (3) for an arbitrary $\mathbf{H}$, and then repeat this operation to recompute the power allocation in successive time instants. Given that in practice we are interested in solving several instances of (3) across time, a learning-based body of work has gained traction in the past years [7 13]. In a nutshell, based on many channel state instances, the idea is to learn a map (i.e., a function approximator) between the channel state matrix $\mathbf{H}$ and the corresponding (approximate) optimal power allocation p. Unlike common neuralnetwork models whose inputs and outputs are often of fixed dimension, the dimension of $\mathbf{H}$ is not necessarily fixed a priori and can indeed change as nodes enter and exit the wireless network. This fact combined with the inherent topological structure present in $\mathbf{H}$ motivates the use of graph neural networks.

Our goal is to combine the advantages of the classical and learning-based paradigms by leveraging the approximate and interpretable solution provided by the classical WMMSE [5] method while enhancing it with the computational run-time efficiency of trained machine learning models. We pursue this synergistic combination under the paradigm of algorithm unfolding, as presented next.

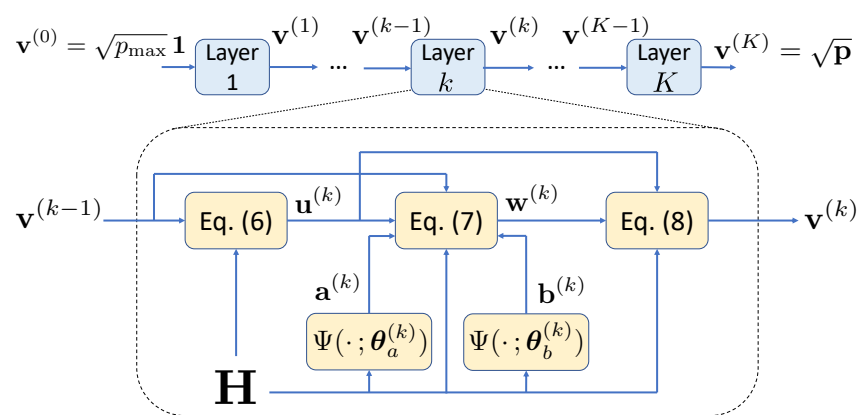

Fig. 1. Schematic diagram of the proposed UWMMSE algorithm. A generic intermediate layer $k$ is detailed.

\section{ALGORITHM UNFOLDING FOR POWER ALLOCATION}

Algorithm unfolding [11,24, 26 refers to the general notion of building a problem-specific neural architecture with layers inspired by an iterative solution to the same problem. In this paper, we develop and evaluate a way to unfold the classical WMMSE [5] method using graph neural networks for power allocation in wireless networks.

We start by introducing the basics of WMMSE. Essential for this classical algorithm is to reformulate (3) as

$$
\begin{aligned}
& \min _{\mathbf{w}, \mathbf{u}, \mathbf{v}} \sum_{i=1}^{M}\left(w_{i} e_{i}-\log w_{i}\right) \\
& \text { s.t. } \quad e_{i}=\left(1-u_{i} h_{i i} v_{i}\right)^{2}+\sigma^{2} u_{i}^{2}+\sum_{i \neq j} u_{i}^{2} h_{i j}^{2} v_{j}^{2}, \quad v_{i}^{2} \leq p_{\max }
\end{aligned}
$$

where the constraints are repeated for all $i$ and $e_{i}$ computes the mean-square error of the signal at node $i$ under the assumption that the transmitted signal $x_{i}$ is independent of the noise $n_{i}$ [cf. [1]].

We say that (4) is equivalent to (3) because it can be shown [5. Thm. 3] that the optimal solution $\left\{\mathbf{w}^{*}, \mathbf{u}^{*}, \mathbf{v}^{*}\right\}$ of the former and that of the latter $\mathbf{p}^{*}$ are related as $\sqrt{\mathbf{p}^{*}}=\mathbf{v}^{*}$, where the square root is applied elementwise. While (4) is non-convex, it is convex in each variable when fixing the other two; this motivates block-coordinate descent, which provides closed-form updates rules to find a local minimum. Our unfolding architecture is inspired by these iteratively applied closed-form equations, which we augment with learnable parameters.

We propose to compute the allocated power as a function of the channel state matrix $\mathbf{p}=\Phi(\mathbf{H} ; \boldsymbol{\Theta})$ through a layered architecture $\Phi$ with trainable weights $\Theta$. More precisely, setting $\mathbf{v}^{(0)}=\sqrt{p_{\max }} \mathbf{1}$, we have that for layers $k=1, \ldots, K$,

$$
\begin{array}{rlrl}
\mathbf{a}^{(k)} & =\Psi\left(\mathbf{H} ; \boldsymbol{\theta}_{a}^{(k)}\right), \quad \mathbf{b}^{(k)}=\Psi\left(\mathbf{H} ; \boldsymbol{\theta}_{b}^{(k)}\right), & \\
u_{i}^{(k)} & =\frac{h_{i i} v_{i}^{(k-1)}}{\sigma^{2}+\sum_{j} h_{i j}^{2} v_{j}^{(k-1)} v_{j}^{(k-1)}}, & & \text { for all } i, \\
w_{i}^{(k)} & =\frac{a_{i}^{(k)}}{1-u_{i}^{(k)} h_{i i} v_{i}^{(k-1)}}+b_{i}^{(k)}, & & \text { for all } i, \\
v_{i}^{(k)} & =\alpha\left(\frac{u_{i}^{(k)} h_{i i} w_{i}^{(k)}}{\sum_{j} h_{j i}^{2} u_{j}^{(k)} u_{j}^{(k)} w_{j}^{(k)}}\right), & & \text { for all } i,
\end{array}
$$

and the output power is determined as $\mathbf{p}=\Phi(\mathbf{H} ; \boldsymbol{\Theta})=\left(\mathbf{v}^{(K)}\right)^{2}$, where the square is applied elementwise. The non-linear function 
$\alpha(z):=[z]_{0}^{\sqrt{p_{\max }}}$ in $(8)$ simply ensures that $v_{i}^{(k)} \in\left[0, \sqrt{p_{\max }}\right]$ by saturating the right-hand side of 8 at these extreme values. This guarantees that the constraint in $(3)$ is satisfied. The trainable parameters $\boldsymbol{\Theta}$ are given by the collection of $\boldsymbol{\theta}_{a}^{(k)}$ and $\boldsymbol{\theta}_{b}^{(k)}$ in $(5)$ for all $K$ layers. Finally, the functions $\Psi$ parametrized by $\Theta$ in $(5)$ are chosen to be graph convolutional networks (GCNs) [17]. A schematic view of our proposed layered architecture is presented in Fig. 1

In the proposed architecture, notice that each layer $k$ as described in (5)-(8) is characterized by five vectors $\mathbf{a}^{(k)}, \mathbf{b}^{(k)}, \mathbf{u}^{(k)}$, $\mathbf{w}^{(k)}, \mathbf{v}^{(k)} \in \mathbb{R}^{M}$. If $\mathbf{a}^{(k)}=\mathbf{1}$ and $\mathbf{b}^{(k)}=\mathbf{0}$, expressions (6)-(8) correspond to the closed-form expressions for block coordinate descent on (4); see [5] for details. In other words, for these values of $\mathbf{a}^{(k)}$ and $\mathbf{b}^{(k)}$, our proposed architecture boils down to a truncated WMMSE with $K$ iterations. In this setting, $\mathbf{u}^{(k)}$ and $\mathbf{v}^{(k)}$ represent receiver and transmitter side variables, respectively, such that $u_{i}^{(k)}$ depends exclusively on the channel states into receiver $r(i)$ whereas $v_{i}^{(k)}$ depends exclusively on the channel states out of transmitter $i$.

A major drawback of WMMSE lies in its high computational and time complexity. This complexity arises because WMMSE requires many iterations of the updates $(6)-(8)$ for convergence. Hence, the objective of the learned variables $\mathbf{a}^{(k)}$ and $\mathbf{b}^{(k)}$ is to accelerate this convergence while maintaining good performance. Intuitively, if we learn a smarter update rule for $\mathbf{w}^{(k)}$ that accelerates its convergence, we can achieve good performance with only a few iterations of WMMSE. Notice that additional learning parameters and more sophisticated functional forms could be included in the updates $(6)-(8)$. However, the learned affine transformation proposed in (7) achieves good performance in practice (see Section (4) while being simple to implement.

Under the natural assumption that the weights $\mathbf{a}^{(k)}$ and $\mathbf{b}^{(k)}$ should depend on the channel state $\mathbf{H}$, we advocate a learning-based method where this dependence is made explicit via the parametric functions $\Psi$ in $(5$ ). For fixed parameters $\Theta$, the allocated power for a channel state $\mathbf{H}$ is given by $\Phi(\mathbf{H} ; \boldsymbol{\Theta})$ and results in a sum-rate utility of $\sum_{i=1}^{M} c_{i}(\Phi(\mathbf{H} ; \boldsymbol{\Theta}), \mathbf{H})$. Hence, we define the loss function

$$
\ell(\boldsymbol{\Theta})=-\mathbb{E}_{\mathbf{H} \sim \mathcal{H}}\left[\sum_{i=1}^{M} c_{i}(\Phi(\mathbf{H} ; \boldsymbol{\Theta}), \mathbf{H})\right] .
$$

Leveraging the facts that GCNs $\Psi(\cdot ; \boldsymbol{\theta})$ in 5 are differentiable with respect to $\boldsymbol{\theta}$ and that we have access to samples of $\mathcal{H}$ (cf. Section2), we seek to minimize 9 through stochastic gradient descent. Notice that we think of UWMMSE as an unsupervised method in the sense that, for training, it requires access to samples of the channel state matrices $\mathbf{H}$ but does not require access to the optimal power allocations (output values) associated with those channels.

Finally, note that the indexing of the $M$ transceiver pairs is arbitrary. Hence, any reasonable power allocation policy should be independent of these indices, which is formally encoded in the notion of permutation equivariance. Given a function $f: \mathbb{R}^{M \times M} \rightarrow \mathbb{R}^{M}$, we say that $f$ is permutation equivariant if $f\left(\mathbf{\Pi} \mathbf{H} \mathbf{\Pi}^{\top}\right)=\Pi \mathbf{\Pi}(\mathbf{H})$ for all $\mathbf{H}$ and all permutation matrices $\Pi$. Naturally, the choice of $\Psi$ in (5) affects the permutation equivariance of our proposed method, as we state next 1

Proposition 1 If $\Psi(\cdot ; \boldsymbol{\theta})$ in 5 is permutation equivariant then the UWMMSE method $\Phi(\cdot ; \boldsymbol{\Theta})$ is also permutation equivariant.

Given that GCNs, our choice for $\Psi$, are known to be permutation equivariant [17], Proposition 1 indicates that UWMMSE is permutation equivariant, as desired.

\footnotetext{
${ }^{1}$ The proof, omitted due to space limitations, can be found in 27 .
}

\section{NUMERICAL EXPERIMENTS}

We simulate a Rayleigh fading channel as a test-bed for our experiments ${ }^{2}$ To that end, we construct a random geometric graph in two dimensions having $M$ transceiver pairs. First, each transmitter $i$ is dropped uniformly at random at locations $\mathbf{t}_{i} \in[-M, M]^{2}$. Then, its paired receiver $r(i)$ is dropped uniformly at random at location $\mathbf{r}_{i} \in\left[\mathbf{t}_{i}-\frac{M}{4}, \mathbf{t}_{i}+\frac{M}{4}\right]^{2}$. Under fading conditions, the channel between a transmitter $i$ and any receiver $r(j)$, at any scheduling instant, is composed of two components $H_{i j}=H_{i j}^{P} H_{i j}^{F}$, where the path gain is given by $H_{i j}^{P}=\left\|\mathbf{t}_{i}-\mathbf{r}_{j}\right\|^{-2.2}$ and the fading coefficient is randomly drawn $H_{i j}^{F} \sim$ Rayleigh(1). These parameter values are approximately representative of realistic path loss and fading in several actual wireless environments. In Section 4.1 we assume that the underlying topology of the network is fixed whereas in Section 4.2 we study UWMMSE's generalization when the size or density of the network changes.

The UWMMSE architecture used is composed of 4 unrolled WMMSE layers with each layer $k$ having two 2-layered GCNs modeling the function $\Psi$ in (5). The hidden layer dimension of all GCNs is set to 5.10000 training iterations are performed per epoch with a maximum of 20 epochs. Batch size is fixed at 64 and learning rate is set to $1 \times 10^{-3}$. We run 100 test iterations with the same batch size. Unless otherwise specified, the network size $M$ is fixed at 20 .

\subsection{Performance comparison}

We compare the performance attained by UWMMSE with that of established baselines in the challenging low-noise regime $[\sigma=$ $2.6 \times 10^{-5}$ in [2] ] where the achievable capacity is highly determined by the interference between users. We choose the following prior-arts for comparison:

1. WMMSE [5] forms the baseline for our experiments. We set a maximum of 100 iterations per sample.

2. Truncated WMMSE (Tr-WMMSE) provides a performance lower bound to UWMMSE. We fix the number of iterations to 4 to match UWMMSE unrollings.

3. $M L P$ [7] is employed in a supervised setting to replicate WMMSE output.

4. REGNN [13] addresses the specific problem of binary power allocation in Rayleigh interference channels.

5. IGCNet [15] addresses the power allocation problem in Gaussian interference channels.

The comparisons are shown in Fig 2 a). As channel states are sampled randomly, there can be significant variation in the utility value for individual samples depending on the respective interference patterns, even under optimal power allocation. The figure reveals that UWMMSE matches the performance of WMMSE. It is also interesting to note that UWMMSE bridges the gap between WMMSE and its truncated version owing to the additional flexibility afforded by learnable parameters. MLP, which learns a functional mapping of the WMMSE output, beats Tr-WMMSE but still falls short of WMMSE by a significant margin. This shows that supervised methods are limited by the quality of their training signals. On the other hand, both REGNN and IGCNet - originally designed and tested in high-noise regimes - prove to be inadequate to match the performance of WMMSE in the more challenging low-noise setting.

\footnotetext{
${ }^{2}$ Code to replicate the numerical experiments here presented can be found at https://github.com/archo48/unrolled-wmmse.git
} 


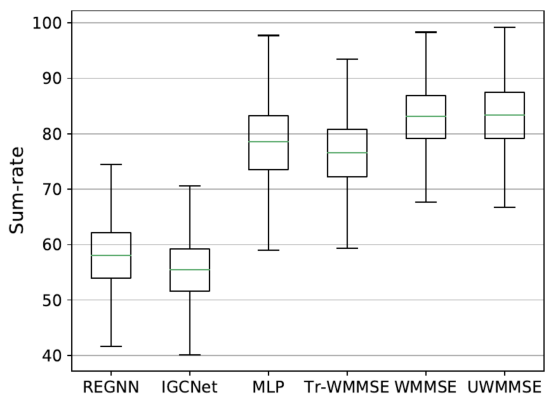

(a)

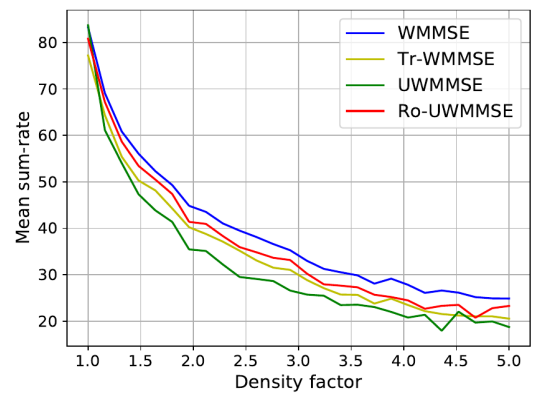

(b)

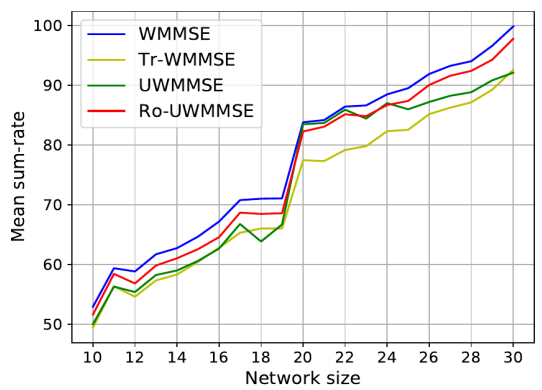

(c)

Fig. 2. Performance and generalization analysis for UWMMSE. (a) Box plots of the achieved utilities for 6400 randomly drawn channel state matrices. (b) Mean sum-rate achieved as density factor $d$ varies in $[1.0,5.0]$, generating denser networks. (c) Mean sum-rate achieved as network size $M$ varies in $\{10, \ldots, 30\}$.

Table 1. Performance and time comparisons of all methods

\begin{tabular}{lccc}
\hline Algorithm & $\begin{array}{c}\text { Train } \\
\text { time }(\mathrm{min})\end{array}$ & $\begin{array}{c}\text { Mean } \\
\text { sum-rate }\end{array}$ & $\begin{array}{c}\text { Test } \\
\text { time }(\mathrm{ms})\end{array}$ \\
\hline WMMSE [5] & - & 82.94 & 16.0 \\
Tr-WMMSE & - & 76.49 & 1.0 \\
MLP [7] & 0.5 & 78.17 & 3.2 \\
REGNN [13] & 15 & 57.92 & 2.5 \\
IGCNet [15] & 5 & 55.30 & 3.0 \\
UWMMSE & 15 & $\mathbf{8 3 . 2 1}$ & $\mathbf{2 . 0}$ \\
\hline
\end{tabular}

In addition to achieving a sum-rate that is close to optimal, it is essential to minimize the time taken for power allocation as the channel states tend to change rapidly. To that end, we provide a computation time comparisor ${ }^{3}$ in Table 1 UWMMSE, which takes close to 2 milliseconds (ms) per sample, is significantly faster than WMMSE which takes around $16 \mathrm{~ms}$ per sample. All the other learning-based methods [7, 13, 15] have a processing time similar to that of UWMMSE, however, none of them achieves the same performance, which is the main advantage of our method over existing algorithms.

\subsection{Generalization to variations in network density and size}

We consider two scenarios in which a wireless network undergoes variations in terms of the density and size of the underlying topology. We simulate a dynamic topology by varying the spatial density of the static network from the previous experiment. A density factor $d$ is used as a control parameter for this experiment. More precisely, for each value of $d$, a transmitter is dropped at location $\mathbf{t}_{i}^{d}=\mathbf{t}_{i} / d$. Its paired receiver $r(i)$, however, is still dropped uniformly at random at location $\mathbf{r}_{i}^{d} \in\left[\mathbf{t}_{i}^{d}-\frac{M}{4}, \mathbf{t}_{i}^{d}+\frac{M}{4}\right]^{2}$. Effectively, the spatial density is varied according to relative positions of the transmitters while maintaining a degree of stochasticity in the receiver positions. We focus on networks that are denser than the original one $(1.0 \leq d \leq 5.0)$ to analyze the challenging case of increasing interference due to geographical proximity; see Fig.2 b).

In this experimental setup, we compare UWMMSE trained on the static network against WMMSE and Tr-WMMSE as baselines. Clearly, there is an approximately constant but moderate gap in performance between WMMSE and UWMMSE for all density values

\footnotetext{
${ }^{3}$ All computations were performed on an Nvidia Quadro T2000 GPU.
}

except $d=1.0$, as UWMMSE achieves limited generalization to unseen network topologies. As an improvement, we introduce a robust version of UWMMSE (Ro-UWMMSE), that is trained on multiple network topologies with varying spatial density $d \in[0.5,5.0]$. As evident in Fig. 2 b), Ro-UWMMSE follows WMMSE performance closely and, importantly, improves upon Tr-WMMSE demonstrating the value of learning even for varying densities.

We now consider a variable-size setup that involves random insertion or deletion of nodes in a wireless network. To that end, a set of transceivers is either removed from the network or a new set of transceivers are added to the network at every scheduling instant. Note that new transceivers are still added in the interval $\mathbf{t}_{i} \in$ $[-M, M]^{2}$ for all $i>M$, where $M$ is the original network size, to avoid any expansion or contraction of the overall area of the multisized topology. Corresponding receivers are dropped $\mathbf{r}_{i} \in\left[\mathbf{t}_{i}-\right.$ $\left.\frac{M}{4}, \mathbf{t}_{i}+\frac{M}{4}\right]^{2}$. Fading coefficients are sampled independently for each individual topology. We evaluate model performance on networks of size $N \in\{10, \ldots, 30\}$ by either removing nodes from the original network $(N<M)$ or adding new unseen nodes to it $(N>M)$, as shown in Fig. 2. $\mathrm{c})$.

Similar to the previous experiment, we compare our method against WMMSE and Tr-WMMSE as baselines and, as expected, UWMMSE performs best on networks that are of the same size as the training samples. To make UWMMSE robust against these variations, we train a robust version Ro-UWMMSE on networks of multiple sizes by randomly generating a batch of networks of size $M \in$ $\{10, \ldots, 30\}$ at each training step. As evident in Fig. 2(c), RoUWMMSE is able to maintain performance that is close to WMMSE and, thus, illustrates the generalization capacity of our methodology.

\section{CONCLUSIONS}

We proposed UWMMSE, a novel neural network based approach to solve the problem of power allocation in wireless networks. The layered architecture of the presented method was derived from the algorithmic unfolding of the classical WMMSE algorithm, thus, UWMMSE naturally incorporates domain-specific elements augmented by trainable components. These components are parameterized by GNNs to account for and leverage the inherent graph representation of communication networks. We have demonstrated that UWMMSE achieves performance comparable to that of WMMSE while being significantly faster than it. Current efforts include analyzing - theoretically and empirically - the model performance under missing, noisy, or even adversarial channel information. 


\section{REFERENCES}

[1] Claude E Shannon, "A mathematical theory of communication," The Bell system technical journal, vol. 27, no. 3, pp. 379-423, 1948.

[2] Holger Boche, Siddharth Naik, and Tansu Alpcan, "Characterization of convex and concave resource allocation problems in interference coupled wireless systems," IEEE Trans. Signal Process., vol. 59, no. 5, pp. 2382-2394, 2011.

[3] Zhi-Quan Luo and Shuzhong Zhang, "Dynamic spectrum management: Complexity and duality," IEEE J. Sel. Topics Signal Process., vol. 2, no. 1, pp. 57-73, 2008.

[4] Meisam Razaviyayn, Mingyi Hong, and Zhi-Quan Luo, "Linear transceiver design for a MIMO interfering broadcast channel achieving max-min fairness," Signal Processing, vol. 93, no. 12, pp. 3327-3340, 2013.

[5] Qingjiang Shi, Meisam Razaviyayn, Zhi-Quan Luo, and Chen $\mathrm{He}$, "An iteratively weighted MMSE approach to distributed sum-utility maximization for a MIMO interfering broadcast channel," IEEE Trans. Signal Process., vol. 59, no. 9, pp. 4331-4340, 2011.

[6] Zhijin Qin, Hao Ye, Geoffrey Ye Li, and Biing-Hwang Fred Juang, "Deep learning in physical layer communications," IEEE Wirel. Commun., vol. 26, no. 2, pp. 93-99, 2019.

[7] Haoran Sun, Xiangyi Chen, Qingjiang Shi, Mingyi Hong, Xiao $\mathrm{Fu}$, and Nicholas D Sidiropoulos, "Learning to optimize: Training deep neural networks for interference management," IEEE Trans. Signal Process., vol. 66, no. 20, pp. 5438-5453, 2018.

[8] Woongsup Lee, Minhoe Kim, and Dong-Ho Cho, "Deep power control: Transmit power control scheme based on convolutional neural network," IEEE Commun. Lett., vol. 22, no. 6, pp. 1276-1279, 2018.

[9] Mark Eisen, Clark Zhang, Luiz FO Chamon, Daniel D Lee, and Alejandro Ribeiro, "Learning optimal resource allocations in wireless systems," IEEE Trans. Signal Process., vol. 67, no. 10, pp. 2775-2790, 2019.

[10] Karol Gregor and Yann LeCun, "Learning fast approximations of sparse coding," in Intl. Conf. Mach. Learn. (ICML), 2010, pp. 399-406.

[11] Vishal Monga, Yuelong Li, and Yonina C Eldar, "Algorithm unrolling: Interpretable, efficient deep learning for signal and image processing," arXiv preprint arXiv:1912.10557, 2019.

[12] Jia Guo and Chenyang Yang, "Structure of deep neural networks with a priori information in wireless tasks," arXiv preprint arXiv:1910.13728, 2019.

[13] Mark Eisen and Alejandro R Ribeiro, "Optimal wireless resource allocation with random edge graph neural networks," IEEE Trans. Signal Process., 2020.

[14] Kota Nakashima, Shotaro Kamiya, Kazuki Ohtsu, Koji Yamamoto, Takayuki Nishio, and Masahiro Morikura, "Deep reinforcement learning-based channel allocation for wireless LANs with graph convolutional networks," arXiv preprint arXiv:1905.07144, 2019.

[15] Yifei Shen, Yuanming Shi, Jun Zhang, and Khaled B Letaief, "A graph neural network approach for scalable wireless power control," arXiv preprint arXiv:1907.08487, 2019.
[16] Joan Bruna, Wojciech Zaremba, Arthur Szlam, and Yann LeCun, "Spectral networks and locally connected networks on graphs," arXiv preprint arXiv:1312.6203, 2013.

[17] Thomas N Kipf and Max Welling, "Semi-supervised classification with graph convolutional networks," in Intl. Conf. Learn. Repres. (ICLR), 2017.

[18] Michaël Defferrard, Xavier Bresson, and Pierre Vandergheynst, "Convolutional neural networks on graphs with fast localized spectral filtering," in Adv. Neural Info. Process. Syst. (NeurIPS), 2016, pp. 3844-3852.

[19] Yaguang Li, Rose Yu, Cyrus Shahabi, and Yan Liu, "Diffusion convolutional recurrent neural network: Data-driven traffic forecasting," arXiv preprint arXiv:1707.01926, 2017.

[20] Fernando Gama, Antonio G Marques, Geert Leus, and Alejandro Ribeiro, "Convolutional neural network architectures for signals supported on graphs," IEEE Trans. Signal Process., vol. 67, no. 4, pp. 1034-1049, 2018.

[21] T. Mitchell Roddenberry and Santiago Segarra, "HodgeNet: Graph neural networks for edge data," in Asilomar Conf. Signals, Systems, and Computers, 2019, pp. 220-224.

[22] Jingkang Yang and Santiago Segarra, "Enhancing geometric deep learning via graph filter deconvolution," in IEEE Global Conf. Signal and Info. Process. (GlobalSIP), 2018, pp. 758762.

[23] Mingyi Hong and Zhi-Quan Luo, "Chapter 8 - Signal Processing and Optimal Resource Allocation for the Interference Channel," in Academic Press Library in Signal Processing: Volume 2, Nicholas D. Sidiropoulos, Fulvio Gini, Rama Chellappa, and Sergios Theodoridis, Eds., vol. 2 of Academic Press Library in Signal Processing, pp. 409 - 469. Elsevier, 2014.

[24] Alexios Balatsoukas-Stimming and Christoph Studer, "Deep unfolding for communications systems: A survey and some new directions," in IEEE Intl. Wrksp. Signal Process. Sys. (SiPS). IEEE, 2019, pp. 266-271.

[25] Risheng Liu, Shichao Cheng, Long Ma, Xin Fan, and Zhongxuan Luo, "Deep proximal unrolling: Algorithmic framework, convergence analysis and applications," IEEE Trans. Image Process., vol. 28, no. 10, pp. 5013-5026, 2019.

[26] Nariman Farsad, Nir Shlezinger, Andrea J Goldsmith, and Yonina C Eldar, "Data-driven symbol detection via modelbased machine learning," arXiv preprint arXiv:2002.07806, 2020.

[27] Arindam Chowdhury, Gunjan Verma, Chirag Rao, Ananthram Swami, and Santiago Segarra, "Unfolding wmmse using graph neural networks for efficient power allocation," arXiv preprint arXiv:2009.10812, 2020. 